\title{
Isomorphism Properties on Strong Fuzzy Hypergraphs
}

\author{
C.Radhika \\ Assistant Professor \\ Kongu Arts and Science \\ College \\ Erode -638 107
}

\author{
C.Radhamani \\ Assistant Professor \\ Kongu Arts and Science \\ College \\ Erode -638 107
}

\author{
S.Suresh \\ Assistant Professor \\ Kongu Arts and Science \\ College \\ Erode -638 107
}

\begin{abstract}
In this paper, the concept of hypergraph was extended to fuzzy hypergraph and paper discusses the connectedness of isomorphic fuzzy hypergraph. Image of strong arc under isomorphism and co-weak isomorphism are studied. Some properties of strong fuzzy hypergraphs are discussed. The image of strong fuzzy hypergraph under isomorphism, coweak isomorphism and weak isomorphism is also studied.
\end{abstract}

\section{Keywords:}

fuzzy hypergraphs, strong arc, complement, isomorphism.

\section{INTRODUCTION:}

Hypergraph theory was introduced in 1960s as a generalization of graph theory. Since then many applications for hypergraph has been developed, for example in engineering, image processing, molecular biology, chemistry etc. In hypergraph more than two vertices may be linked so that the hyperedge of a hypergraph are subsets of vertex sets. A standard reference of this theory is due to C.Berge[1].

Rosenfield (1975) introduced the notion of fuzzy graphs and several graph theoretic concepts such as paths, cycles and connectedness. Moderson.J.N and P.S.Nair [5] gave the definition for fuzzy hypergraph. A.Nagoorgani and J.Malarvizhi [7] introduced isomorphism properties on strong fuzzy graphs and also discussed about complements of fuzzy graph. C.Radhamani and C.Radhika [8] introduced isomorphism on fuzzy hypergraph. Further in this paper authors proposed to introduce isomorphism properties on strong fuzzy hypergraphs.

In this paper we have discussed some isomorphism properties fuzzy hypergraphs with reference to strong arc in fuzzy hypergraph, strong fuzzy hypergraphs and also about complement of fuzzy hypergraphs.

\section{PRELIMINARIES}

\section{Definition[5]}

Let $X$ be a finite set and let $\varepsilon$ be a finite family of non trivial fuzzy subsets of $X$ such that

$\mathrm{X}=\bigcup_{\mu_{i}} \operatorname{supp} \mu_{i}(x)$. Then the pair $H:(X, \varepsilon)$ is called a fuzzy hypergraph and $\varepsilon=\left\{E_{1}, E_{2}, \ldots E_{n}\right\}$ is called the collection of edge sets of $H$.

\section{Note}

A fuzzy hypergraph $H$ with underlying set $X$ is $H:\left(X, \mu_{i}, \rho\right) \quad$ where $\quad \mu_{i}: X \rightarrow[0,1]$ are fuzzysubsets, $\rho: \varepsilon \rightarrow[0,1]$ is a fuzzy relation on the fuzzy subsets $\mu_{i}$

$$
\begin{aligned}
& \rho\left(\left\{x_{1}, x_{2}, \ldots \ldots . x_{r}\right\}\right) \leq\left[\wedge \mu_{i}\left(x_{2}\right)\right] \wedge\left[\mu_{i}\left(x_{2}\right)\right] \wedge \ldots . . \wedge\left[\mu_{i}\left(x_{r}\right)\right] \\
& \forall\left\{x_{1}, x_{2}, \ldots . . x_{r}\right\}=E_{i} \subset X
\end{aligned}
$$

\section{Definition 2.1}

A fuzzy hypergraph $H$ with $X$ as the underlying set $H$ : $\left(X, \mu_{i}, \rho\right)$ where

$$
\begin{aligned}
& \mu_{i}: E_{i} \rightarrow[0,1] \quad \ni X=\bigcup_{\mu_{i}} \operatorname{supp} \mu_{i}(x) \\
& \text { and } \rho: \varepsilon \rightarrow[0,1] \ni \rho\left(E_{i}\right) \leq \wedge \mu_{i}(x) \forall x \in E_{i}
\end{aligned}
$$

The underlying crisp hypergraph of the fuzzy hypergraph $H:\left(X, \mu_{i}, \rho\right)$ is denoted by $H^{*}:\left(X, \mu_{i}^{*}, \rho^{*}\right)$ where $\mu_{i}^{*}=\operatorname{supp} \mu_{i}$ and $\rho^{*}=\operatorname{supp} \rho$

\section{Note:}

Throughout this paper $H:\left(X, \mu_{i}, \rho\right)$ and $H^{\prime}:\left(X, \mu_{i}^{\prime}, \rho^{\prime}\right)$ are taken as fuzzy hypergraphs with underlying sets $X$ and $X^{\prime}$ respectively.

\section{Definition 2.2}

A path $\sigma$ in a fuzzy hypergraph $H:\left(X, \mu_{i}, \rho\right)$ is a sequence of distinct nodes

$$
\begin{aligned}
& v_{0}, v_{1}, \ldots \ldots \ldots v_{n} \ni \mu_{i}\left(v_{j}\right)>0 \\
& \text { and } \mu_{i+1}\left(v_{j}\right)>0 \quad j=1,2,3, \ldots \ldots . n, i=1,2,3, \ldots \ldots . n
\end{aligned}
$$

Here $\mathrm{n}$ is called the length of the path. The consecutive pairs $\left(v_{i-1}, v_{i}\right)$ are called arcs of the path.

\section{Definition 2.3}

If $x, y$ are nodes in the fuzzy hypergraph $H$ and if they are connected by means of a path, then the strength of that path is defined as

$$
\sigma(x, y)=\stackrel{\hat{j}_{j=1}^{n}}{\wedge}\left(E_{i}\right) \text { for } x \in E_{1}, y \in E_{n} \text {. It is the }
$$
strength of the weakest arc.

If $x, y$ are connected by means of paths of length $\mathrm{k}$, then 


$$
\begin{aligned}
& \sigma^{k}(x, y)=\left\{\rho\left(E_{1}\right) \wedge \rho\left(E_{2}\right) \ldots \ldots . . \wedge \rho\left(E_{k}\right) / x \in E_{1},\right. \\
& y \in E_{k}, x=\left\{x_{1}, x_{2}, \ldots \ldots x_{k-1}, y \in X\right\}
\end{aligned}
$$

If $x, y \in X$, the strength of connectedness between $\mathrm{x}$ and $\mathrm{y}$ is $\sigma^{\infty}(x, y)=\sup .\left\{\sigma^{k}(x, y) / k=1,2,3 \ldots \ldots ..\right\}$

\section{Definition 2.4}

A fuzzy hypergraph $H$ is connected if $\sigma^{\infty}(x, y)>0 \forall x, y \in \mu_{i}^{*}$.

\section{Definition 2.5}

A fuzzy hypergraph $H$ is said to be a strong or effective fuzzy hypergraph if

$\rho\left(x_{1}, x_{2}, \ldots \ldots x_{r}\right)=\underset{j=1}{r}\left[\wedge \mu_{i}\left(x_{j}\right) \forall\left\{x_{1}, x_{2}, \ldots ., x_{r}\right\}=E_{i} \subset X\right.$

in $\rho^{*}$

\section{Definition 2.6}

A fuzzy hyper graph $H$ is said to be a complete fuzzy hypergraph if

$$
\rho\left(x_{1}, x_{2}, \ldots . . x_{r}\right)=\stackrel{r}{\wedge}\left[\wedge \mu_{i}\left(x_{j}\right)\right] \forall x \in E_{i} \text { in } \mu_{i}{ }^{*}
$$

\section{Definition 2.7}

An $\operatorname{arc}(x, y)$ in a fuzzy hypergraph is said to be a strong arc if $\sigma(x, y) \geq \sigma^{\infty}(x, y)$.

A node $x$ is said to be an isolated node if $x$ does not combine with any vertex in any edge in the fuzzy hypergraph.

\section{Definition 2.8}

A homomorphism of fuzzy hypergraph $h: H \rightarrow H^{\prime}$ is a map $h: X \rightarrow X^{\prime}$ which satisfies

$$
\begin{aligned}
& \wedge \mu_{i}(x) \leq \wedge \mu_{i}^{\prime}[h(x)] \forall x \in X \\
& \text { and } \rho\left(E_{i}\right)=\rho^{\prime}\left[h\left(E_{i}\right)\right] \forall E_{i} \in \varepsilon \\
& \rho\left(x_{1}, x_{2}, \ldots ., x_{r}\right)=\rho^{\prime}\left[h\left(x_{1}\right), \ldots \ldots h\left(x_{r}\right)\right] \quad \forall\left\{x_{1}, x_{2}, \ldots ., x\right\}=E_{i} \subset X
\end{aligned}
$$

\section{Definition 2.9}

A weak isomorphism $h: H \rightarrow H^{\prime}$ is a map $h: X \rightarrow X^{\prime}$ such that the bijective homomorphism which satisfies $\wedge \mu_{i}(x)=\wedge \mu_{i}^{\prime}[h(x)] \forall x \in X$.

\section{Definition 2.10}

A co-weak isomorphism $h: H \rightarrow H^{\prime}$ is a map $h: X \rightarrow X^{\prime}$ is a bijective homomorphism that satisfies

$$
\begin{aligned}
& \rho\left(x_{1}, x_{2}, \ldots ., x_{r}\right)=\rho^{\prime}\left[h\left(x_{1}\right), \ldots \ldots . h\left(x_{r}\right)\right] \\
& \forall\left\{x_{1}, x_{2}, \ldots ., x_{r}\right\}=E_{i} \subset X
\end{aligned}
$$

\section{Definition 2.11}

An isomorphism is a map $h: X \rightarrow X^{\prime}$ which is a bijective homomorphism that satisfies

$$
\begin{aligned}
& \wedge \mu_{i}(x)=\wedge \mu_{i}^{\prime}[h(x)] \forall x \in X \text { and } \\
& \rho\left(x_{1}, x_{2}, \ldots ., x_{r}\right)=\rho^{\prime}\left[h\left(x_{1}\right), \ldots \ldots . . h\left(x_{r}\right)\right] \\
& \forall\left\{x_{1}, x_{2}, \ldots ., x_{r}\right\}=E_{i} \subset X
\end{aligned}
$$

\section{Isomorphism and Strong arcs in fuzzy hypergraphs}

\section{Theorem3.1}

If $H$ is isomorphic to $H^{\prime}$, then $H$ is connected iff $H^{\prime}$ is also connected.

Proof:

$H$ is isomorphic to $H^{\prime}$, Then there exists an isomorphism $h: H \rightarrow H^{\prime}$ which is a bijective homomorphism satisfying

$$
\begin{aligned}
\sigma^{\infty}(x, y)= & \sup \left\{\sigma^{k}(x, y) / k=1,2,3 \ldots \ldots .\right\} \\
= & \sup \left\{\wedge \rho\left(E_{i}\right) / i=1,2, \ldots . k \quad x \in E_{1},\right. \\
& \left.y \in E_{k}, x, x_{1}, x_{2}, \ldots \ldots x_{k-1}, y \in X\right\} \\
= & \sup \left\{\wedge \rho^{\prime}\left(E_{i}^{\prime}\right) / i=1,2, \ldots k h(x) \in E_{1}^{\prime},\right. \\
& \quad h(y) \in E_{k}^{\prime}, h(x), h\left(x_{1}\right), h\left(x_{2}\right) \ldots . . h\left(x_{k-1}, h(y) \in X^{\prime}\right\} \\
= & \sup \left[\sigma^{\prime k}[h(x), h(y)] / k=1,2 \ldots .\right\} \\
= & \sigma^{\prime \infty}[h(x), h(y)]
\end{aligned}
$$

Therefore $H$ is isomorphic to $H^{\prime}$ implies $\sigma^{\infty}(x, y)=\sigma^{\prime \infty}(h(x), h(y)) \forall x, y \in X$

$\mathrm{H}$ is connected

$$
\begin{aligned}
& \text { iff } \sigma^{\infty}(x, y)>0 \\
& \text { iff } \sigma^{. \infty}(h(x), h(y))>0 \\
& \text { iff } H^{\prime} \text { is connected }
\end{aligned}
$$

Hence the proof.

\section{Theorem 3.2}

If $H$ is isomorphic to $H^{\prime}$, then an $\operatorname{arc}$ in $H$ is strong iff the corresponding image arc in $H$ 'is also strong.

\section{Proof:}

Let $(x, y)$ be a strong arc in $H$.

$$
\Rightarrow \sigma(x, y) \geq \sigma^{\infty}(x, y)
$$

As $h$ is an isomorphism between $H$ and $H^{\prime}$ $\sigma(x, y)=\sigma^{\prime}[h(x), h(y)]$

$$
\begin{aligned}
\therefore \sigma^{\prime}[h(x), h(y)] & \geq \sigma^{\infty}(x, y) \\
= & \sigma^{\prime \infty}(h(x), h(y))
\end{aligned}
$$

(Since $H$ is isomorphic to $H^{\prime}$ ) which implies $(h(x), h(y))$ is a strong arc in $H^{\prime}$

Conversely, by bijective and isomorphism property of $h$, strong arc in $H^{\prime}$ implies its pre-image in $H$ is also strong. 


\section{Remark 3.11}

The results of the above two theorems are true even if the fuzzy hypergraph $H$ is co weak isomorphic to $H^{\prime}$, as the weights of the edges are preserved.

\section{Remark 3.12}

If $H$ isa connected fuzzy hypergraph that is weak isomorphic with $H^{\prime}$, then $H^{\prime}$ is also connected. But the converse is not true. In the following example $H$ and $H^{\prime}$ are two fuzzy hypergraphs such that $H$ is weak isomorphic to $H^{\prime}$. Here $H$ is not connected but $H^{\prime}$ is connected.

Table : 1

$\begin{array}{cccc}H & E_{1} & E_{2} & E_{3} \\ & & & \\ a & 1 / 4 & 0 & 0 \\ b & 1 / 3 & 1 / 2 & 0 \\ c & 0 & 1 / 4 & 0 \\ d & 0 & 0 & 1 / 5\end{array}$

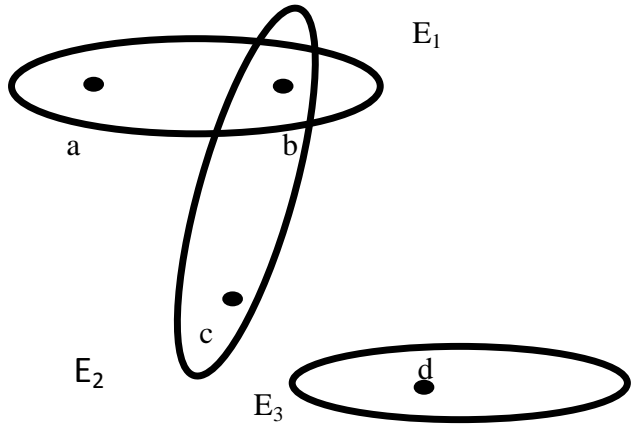

Figure :1 $(H)$

Table : 2

$$
\begin{array}{cccc}
H^{\prime} & E_{1}{ }^{\prime} & E_{2}{ }^{\prime} & E_{3}{ }^{\prime} \\
& & & \\
a^{\prime} & 0 & 1 / 4 & 0 \\
b^{\prime} & 0 & 1 / 3 & 1 / 2 \\
c^{\prime} & 1 / 4 & 0 & 0 \\
d^{\prime} & 1 / 5 & 0 & 1 / 3
\end{array}
$$

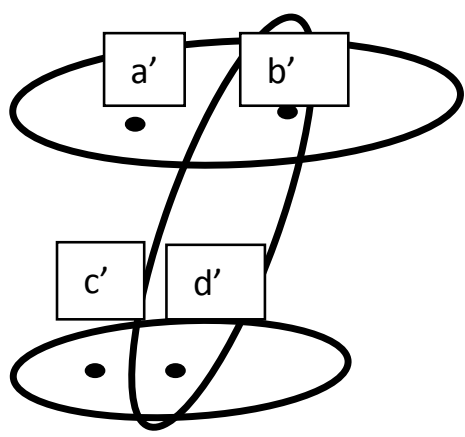

Figure :2 $\left(H^{\prime}\right)$

$$
\begin{aligned}
& \rho\left(E_{1}\right)=1 / 5, \rho\left(E_{2}\right)=1 / 6, \rho\left(E_{3}\right)=1 / 5 \\
& \rho^{\prime}\left(E_{1}\right)=1 / 5, \rho^{\prime}\left(E_{2}\right)=1 / 6 \rho^{\prime}\left(E_{3}\right)=1 / 5
\end{aligned}
$$

Remark 3.13

Under weak isomorphism, strong arcs need not have their image as strong arcs and vice-versa.

\section{Example:}

Here $H$ is weak isomorphic to $H^{\prime}$ under the map $h: X \rightarrow X^{\prime}$ defined as

$h(u)=u^{\prime}, h(w)=w^{\prime}, h(x)=x^{\prime}, h(y)=y^{\prime}$

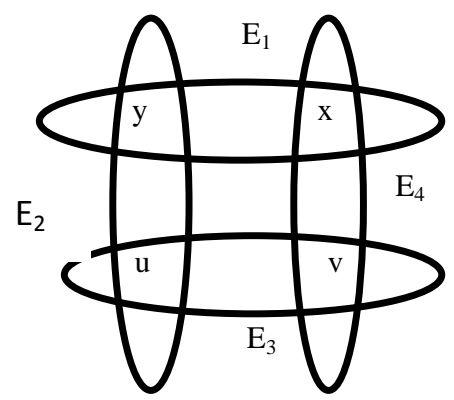

Figure :3 (H)

$\rho\left(E_{1}\right)=1 / 7, \rho\left(E_{2}\right)=1 / 6, \rho\left(E_{3}\right)=1 / 7, \rho\left(E_{4}\right)=1 / 5$

Table: 3 and 4

$\begin{array}{ccccc}H & E_{1} & E_{2} & E_{3} & E_{4} \\ & 1 / 3 & 0 & 0 & 1 / 4 \\ x & 1 & 1 & 0 & 0 \\ y & 1 / 2 & 1 & 0 \\ u & 0 & 1 & 1 & \end{array}$



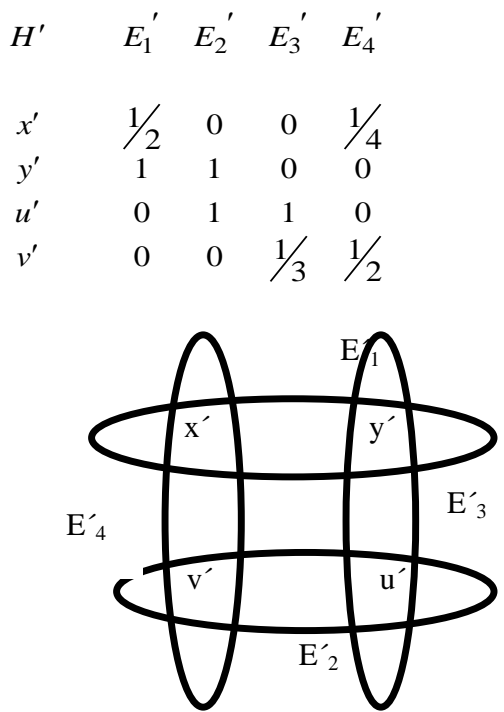

Figure : $4\left(H^{\prime}\right)$

$$
\begin{aligned}
& \rho\left(E_{1}{ }^{\prime}\right)=1 / 7, \rho\left(E_{2}{ }^{\prime}\right)=1 / 3, \\
& \rho\left(E_{3}{ }^{\prime}\right)=1 / 4, \rho\left(E_{4}{ }^{\prime}\right)=1 / 6 \\
& \sigma^{1}(x, y)=\stackrel{4}{\wedge} \rho\left(E_{i}\right)=\frac{1}{7} \\
& \sigma^{k}(x, y)=\left\{\sigma\left(x, y_{1}\right) \wedge \sigma\left(y_{1}, y_{2}\right) \wedge \ldots \ldots . \wedge \sigma\left(y_{k-1}, y\right)\right\} \\
& \sigma^{2}(x, y)=\{\sigma(x, v) \wedge \sigma(v, u) \wedge \sigma(u, y)\}=\frac{1}{7} \\
& \sigma^{\infty}(x, y)=\sup \left\{\sigma^{k}(x, y) / k=1,2\right\} \\
& =\sup \{1 / 7,1 / 7\}=1 / 7 \\
& \sigma(x, y)=\stackrel{\wedge}{i=1}_{\wedge}^{n} \rho\left(E_{i}\right)=\frac{1}{7} \\
& \sigma(x, y)=\sigma^{\infty}(x, y) .
\end{aligned}
$$

Therefore $(x, y)$ is strong in $H$.

$$
\begin{aligned}
& \sigma^{\prime 1}\left(x^{\prime}, y^{\prime}\right)=\frac{1}{7} \\
& \begin{aligned}
\sigma^{\prime}\left(x^{\prime}, y^{\prime}\right) & =\left\{\sigma^{\prime}\left(x^{\prime}, y_{1}^{\prime}\right) \wedge \sigma^{\prime}\left(y_{1}^{\prime}, y_{2}^{\prime}\right) \wedge \ldots \ldots . \wedge \sigma^{\prime}\left(y_{k-1}, y^{\prime}\right)\right\} \\
\sigma^{\prime 2}\left(x^{\prime}, y^{\prime}\right) & =\left\{\sigma^{\prime}\left(x^{\prime}, v^{\prime}\right) \wedge \sigma^{\prime}\left(v^{\prime}, u^{\prime}\right) \wedge \sigma^{\prime}\left(u^{\prime}, y^{\prime}\right)\right\}=\frac{1}{6} \\
\sigma^{\prime \infty}\left(x^{\prime}, y^{\prime}\right) & =\sup \left\{\sigma^{\prime k}\left(x^{\prime}, y^{\prime}\right) / k=1,2\right\} \\
= & \sup \{1 / 7,1 / 6\}=1 / 6 \\
\sigma^{\prime}\left(x^{\prime}, y^{\prime}\right) & =\frac{1}{7} \\
\sigma^{\prime}\left(x^{\prime}, y^{\prime}\right) \prec & \sigma^{\prime \infty}\left(x^{\prime}, y^{\prime}\right) .
\end{aligned}
\end{aligned}
$$

Therefore $\left(x^{\prime}, y^{\prime}\right)$ is not strong in $H^{\prime}$.

\section{Isomorphism and Strong fuzzy hypergraphs}

\section{Theorem: 4.1}

If $H$ is a connected strong fuzzy hypergraph, then every arc in $H$ is a strong arc.

\section{Proof:}

Let $H$ be a strong fuzzy hypergraph

$\therefore \rho\left(x_{1}, x_{2}, \ldots \ldots . . x_{r}\right)=\left(\wedge \mu_{i}\left(x_{j}\right)\right) \quad j=1,2, \ldots . r$

$\forall\left(x_{1}, x_{2}, \ldots . x_{r}\right)=E_{i} \subset \rho^{*}$

\section{Case: 1}

If $(x, y)$ is the only hyper arc connecting the nodes $x$ and $y, \quad \sigma(x, y)=\sigma^{\infty}(x, y)$

Case: 2 If there are paths connecting the nodes $x$ and y other than the edge $(x, y)$ then consider the arbitrary path $\sigma: x=x_{0}, x_{1}, x_{2}, \ldots \ldots . . x_{n}=y$

The strength of the path $\sigma(x, y)=\hat{j=1}_{j=1}^{n} \rho\left(E_{i}\right)$

$$
\begin{aligned}
& =\wedge_{i=1}^{n}\left(\stackrel{m}{\wedge} \mu_{j=1}\left(x_{k}\right)\right) \\
& =\min \mu_{i}\left(x_{j}\right) \\
& \leq\left[\wedge \mu_{i}(x)\right] \wedge\left[\wedge \mu_{i}(y)\right]
\end{aligned}
$$

$\sigma^{\infty}(x, y)=\sup \{$ strengthf all pathsconnectingxand $y\}$

$$
=\sup \left\{\wedge \rho\left(E_{i}\right)\right\}
$$$$
\leq\left[\wedge \mu_{i}(x)\right] \wedge\left[\wedge \mu_{i}(y)\right]
$$$$
=\sigma(x, y)
$$

$\sigma^{\infty}(x, y) \leq \sigma(x, y)$

Hence $(x, y)$ is strong arc in $H$ by both cases

\section{Theorem: 4.2}

If $H$ is isomorphic to $H^{\prime}$, then $H$ is a strong fuzzy hypergraph iff $H^{\prime}$ is also strong fuzzy hyper graph.

\section{Proof:}

$$
H \text { is isomorphic to } H^{\prime}
$$

$\Rightarrow$ There exists an isomorphism

$h: H \rightarrow H^{\prime}$, which is bijective that satisfies

$$
\begin{aligned}
& \wedge\left[\mu_{i}(x)\right]=\wedge\left[\mu_{i}^{\prime}(h(x)] \forall x \in X\right. \text { and } \\
& \rho\left(x_{1}, x_{2}, \ldots . x_{r}\right)=\rho^{\prime}\left[h\left(x_{1}\right), h\left(x_{2}\right) \ldots \ldots . . . h\left(x_{r}\right)\right] \forall x_{1}, x_{2}, \ldots . . x_{r}=E_{i} \subset X
\end{aligned}
$$


This implies $\mathrm{H}^{\prime}$ is a strong fuzzy hypergraph

$$
\begin{aligned}
& \text { iff } \rho\left(x_{1}, x_{2}, \ldots \ldots . . . x_{r}\right)=\wedge\left(\hat{i=1}_{i=1}^{r} \mu_{i}\left(x_{j}\right)\right) \quad j=1,2, \ldots . r \\
& \forall\left(x_{1}, x_{2}, \ldots . x_{r}\right)=E_{i} \subset \rho^{*} \\
& \text { iff } \rho^{\prime}\left(h\left(x_{1}\right), h\left(x_{2}\right), \ldots \ldots . . . h\left(x_{r}\right)\right)=\wedge\left[\wedge \mu_{i}^{\prime}\left(h\left(x_{i}\right)\right] \text { in } \rho^{*}\right. \\
& \text { iff } \rho^{\prime}\left(x_{1}^{\prime}, x_{2}, \ldots \ldots \ldots x_{r}^{\prime}\right)=\wedge\left[\wedge \mu_{i}^{\prime}\left(x_{j}^{\prime}\right)\right] \forall\left(x_{1}^{\prime}, x_{2}^{\prime} \ldots x_{r}^{\prime}\right)=E_{i}^{\prime} \subset X^{\prime}
\end{aligned}
$$

(i.e) iff $H^{\prime}$ is a strong fuzzy hypergraph. Similarly we can prove the converse part

\section{Theorem: 4.3}

If $H$ is co-weak isomorphic with a strong fuzzy hypergraph $H^{\prime}$. Then $H$ is also a strong fuzzy hypergraph

\section{Proof:}

As $H$ is co-weak isomorphic with $H^{\prime}$, there exists a co-weak isomorphism $\boldsymbol{h}: \boldsymbol{H} \rightarrow \boldsymbol{H}^{\prime}$, which is bijective that satisfies

$$
\begin{aligned}
& \wedge\left[\mu_{i}(x)\right] \leq \wedge\left[\mu_{i}^{\prime}(h(x)] \forall x \in X\right. \text { and } \\
& \rho\left(x_{1}, x_{2}, \ldots . . x_{r}\right)=\rho^{\prime}\left[h\left(x_{1}\right), h\left(x_{2}\right) \ldots \ldots . . . h\left(x_{r}\right)\right] \\
& \forall x_{1}, x_{2}, \ldots \ldots x_{r}=E_{i} \subset X
\end{aligned}
$$

Since $H^{\prime}$ is a strong fuzzy hypergraph

$$
\begin{aligned}
& {\left[\wedge \mu ^ { \prime } [ h ( x _ { 1 } ) ] \wedge \left[\wedge \mu^{\prime}\left[h\left(x_{2}\right)\right] \wedge \ldots . . \wedge\left[\wedge \mu^{\prime}\left[h\left(x_{r}\right)\right]\right.\right.\right.} \\
& =\rho^{\prime}\left[h\left(x_{1}\right), h\left(x_{2}\right) \ldots \ldots . . h\left(x_{r}\right)\right] \\
& =\rho\left(x_{1}, x_{2}, \ldots \ldots . x_{r}\right) \\
& \leq\left[\wedge \mu\left(x_{1}\right)\right] \wedge\left[\wedge \mu\left(x_{2}\right)\right] \ldots . . \wedge\left[\wedge \mu\left(x_{r}\right)\right] \\
& \leq\left[\wedge \mu^{\prime} h\left(x_{1}\right)\right] \wedge\left[\wedge \mu^{\prime} h\left(x_{2}\right)\right] \ldots . . \wedge\left[\wedge \mu^{\prime} h\left(x_{r}\right)\right] \\
& \rho\left(x_{1}, x_{2}, \ldots \ldots . . x_{r}\right)=\left[\wedge \mu\left(x_{1}\right)\right] \wedge\left[\wedge \mu\left(x_{2}\right)\right] \ldots . \wedge\left[\wedge \mu\left(x_{r}\right)\right] \forall E_{i} \subset X \text { in } \rho^{*}
\end{aligned}
$$

Therefore $H$ is a strong fuzzy hypergraph

\section{Remark 4.11}

Under co-weak isomorphism of $H$ with $H^{\prime}$, strongness of $H$ need not imply that of $H^{\prime}$.

\section{Remark 4.12}

When $H$ is weak isomorphic to $H^{\prime}$, then strong arcs of one fuzzy hypergraph need not imply the strongness of the other.

\section{CONCLUSION}

A brief review of the theory of fuzzy hypergraphs has been done and the concept of isomorphism,weak isomorphism, co-weak isomorphism on strong fuzzy hypergraphs are introduced and some interesting properties like strong arc, connectedness, path are proved. Further research can be carried to have applications in the field of pattern recognition, clustering, image retrieval, medicine using the concept of fuzzy hypergraphs etc..

\section{REFERENCES}

[1]. Berge.C., 1970,Graphes et Hypergraphes. Dunod , Paris,(Graphs and Hypergraphs, NorthHolland,Amsterdam, 1973, revised translation).

[2]. Bhutani, K.R., 1989, On Automorphism of Fuzzy graphs, Pattern Recognition Letter 9: 159-162.

[3]. Bhattacharya. P,1987, Some Remarks on fuzzy graphs, pattern Recognition Letter 6: 297-302.

[4]. F. Harary, 1969, "Graph theory", Addison Wesley,Reading Mass .

[5]. Moderson, J.N, and P .S. Nair, 1998, Fuzzy Graphs and Fuzzy Hypergraphs Physica verlag, Heidelberg ; Second Edition 2001.

[6].. Nagoorgani.A and Malarvizhi.J , 2008 Isomorphism onFuzzy Graph, International journal of computational and mathematicalsciences, 190-196.

[7]. A.Nagoorgani and J.Malarvizhi., 2009 Isomorphism properties on strong Fuzzy Graphs. International journal of Algorithms, computing and mathematics, Volume 2, February.

[8]. Radhamani.CandRadhika.C,September 2012, Isomorphism on Fuzzy Hypergraphs International journalof Scientific and Research.Volume-II

[9]. Sunitha.M.S and Vijayakumar.A ,2002Complement of Fuzzy graphs, Indian J.Pure and Applied maths 33 [9]; 1451-1464,September. 